\title{
CARCINOMA MUCOEPIDERMOIDE EN PALADAR, REPORTE DE CASO
}

\section{MUCOEPIDERMOID CARCINOMA IN PALATE, CASE REPORT}

\author{
Aguilar José David,*1,Delgado María Augusta, ${ }^{2}$,Terreros Andrea Catalina. ${ }^{3}$ \\ ${ }^{1}$ Cirujano y Patólogo Bucal, Docente de Patología, Histoembriología y Cirugía de la Universidad Católica de Cuenca, Sede \\ Azogues. Ecuador. \\ 2 Odontopediatra y Ortodoncista. Docente de Odontopediatría de la Universidad Católica de Cuenca, Sede Azogues. \\ Ecuador. \\ 3 Odontopediatra, Mgs Gerencia en Salud. Investigadora independiente. Ecuador. \\ *jdaguilarm@ucacue.edu.ec
}

\begin{abstract}
Resumen
El Carcinoma Mucoepidermoide (CME) es la neoplasia maligna más común de las glándulas salivales y se presenta con mayor frecuencia a nivel de glándulas salivales mayores siendo la Parótida la más afectada y a nivel de las glándulas salivales menores se presenta con mayor frecuencia en el paladar. Este artículo presenta el caso de un paciente masculino de 30 años de edad sin antecedentes médicos de importancia, quien acude al servicio de Estomatología del Hospital Central de la Policía Nacional (Bogotá-Colombia), refiriendo presencia de lesión asintomática en paladar blando en 5 meses de evolución. La cual fue diagnosticada como Carcinoma Mucoepidermoide de bajo grado. El objetivo fue presentar un caso de CME en el paladar blando para colaborar con los estomatólogos clínicos para direccionar de mejor manera el diagnóstico temprano de lesiones neoplásicas de origen glandular, concluyendo que ante la presencia de lesiones en cavidad bucal es importante contar con una amplia variedad de impresiones diagnósticas y realizar biopsia para establecer diagnóstico definitivo.
\end{abstract}

Palabras clave: Carcinoma mucoepidermoide, glándulas salivales, neoplasia, tumor.

\begin{abstract}
Mucoepidermoid carcinoma (CME) is the most common malignancy of salivary glands and occurs most often at the level of major salivary glands, being the most affected parotid. At minor salivary glands level occurs most frequently on the palate. This article presents the case of a 30 years old male patient with no medical history of importance, who attends the service of Stomatology on Central Hospital of the National Police (Bogota, Colombia), referring presence of asymptomatic lesion on soft palate with 5 months of evolution. Which it was diagnosed as low-grade carcinoma Mucoepidermoid. The objective was to present a case of CME in the soft palate to collaborate with clinical dentists to guide the early diagnosis of neoplastic lesions of glandular origin, concluding that the presence of lesions in the oral cavity is important to have a wide variety of diagnostic impressions and perform biopsy and histopathological studies to establish a definitive diagnosis.
\end{abstract}

Key words: Mucoepidermoid carcinoma, neoplasia, salivary glands, tumor.

\section{INTRODUCCIÓN}

Los tumores de las glándulas salivales representan entre el 2 y $3 \%$ de las neoplasias de cabeza y cuello. ${ }^{1}$ Siendo el Carcinoma Mucoepidermoide (CME) la neoplasia maligna más común de las glándulas salivales, ${ }^{1-3}$ y comprende aproximadamente el 10-15\% de todas las neoplasias de las glándulas salivales y el $30 \%$ de las neoplasias malignas. ${ }^{4}$ Este tumor se presenta con más frecuencia en la glándula parótida, entre el 60 y $90 \%$ de los casos y representa del 15 al $21 \%$ de los tumores de las glándulas salivales menores, siendo la localización más frecuente en el paladar en un 35\%. ${ }^{5,6}$ La única alteración genética mayormente descrita hasta el momento en relación a $\mathrm{CME}$ es la translocación recíproca recurrente cromosómica t $(11 ; 19)$ (q21; p13), la cual provoca la fusión génica CRTC1-MAML2. ${ }^{2}$ Clínicamente se puede presentar como un aumento de volumen que evoluciona lentamente y sin generar sintomatología, o puede presentarse en ocasiones como una úlcera acompañada de parestesia. $^{7}$ Además debido a los productos de degradación vascular que contienen los quistes dentro de la neoplasia, son comúnmente 
confundidos con Mucocele. ${ }^{7}$ Estructuralmente un CME está compuesto por varios tipos de células como secretoras de mucina, células basaloides, intermedias y epidermoides, que presenta frecuentemente un componente quístico; pueden ser categorizados en tres grados histológicos según la graduación tumoral de Auclair (Tabla 1). , $^{1}$

\begin{tabular}{lc}
\hline Parámetros & Puntaje \\
\hline Componente intiaquístico $<20 \%$ & +2 \\
Invasión neural & +2 \\
Necrosis & +3 \\
4 mitosis/1 0 campos a mayor aumento & +3 \\
A napias i a & +4 \\
Grado & \\
Bajo & \\
Intermedio & $0-4$ \\
Alto & $5-6$ \\
\hline
\end{tabular}

Tabla 1. Categorización del CME.

Los parámetros clínicos convencionales, tales como, la edad y la localización se encuentran entre los factores pronósticos más importantes, junto con los criterios de clasificación histopatológicos. , $^{1,8}$

La terapéutica principal en CME así como en la mayoría de neoplasias malignas de glándulas salivales, es la resección quirúrgica. No se tiene evidencia de que la radioterapia o la quimioterapia mejoren el pronóstico postoperatorio; sin embargo Sadner y cols. recomiendan la cirugía seguida de radioterapia, siendo este tratamiento reservado para CME de alto grado de malignidad, cuya metástasis se realiza de forma más temprana. ${ }^{8}$

En cuanto a la sobrevida de este tumor a 5 años es del $95 \%$ y se asocia con los tumores de bajo grado de malignidad. Por otro lado, para los CME de alto grado la tasa de sobrevida a 5 años es de $40 \%$, siendo en estos casos el $25 \%$ o menos la proporción más alta de resolución, en cuanto a su tasa de recidiva es menor al $10 \%$ en los casos de tumores bien diferenciados. ${ }^{9}$

El objetivo de este trabajo es presentar un caso de CME en paladar blando para colaborar con los estomatólogos clínicos para direccionar de mejor manera el diagnóstico temprano de lesiones neoplásicas de origen glandular.

\section{REPORTE DE CASO}

Paciente masculino de 30 años de edad sin antecedentes médicos de importancia, quien acudió al servicio de Estomatología del Hospital Central de la Policía Nacional (Bogotá-

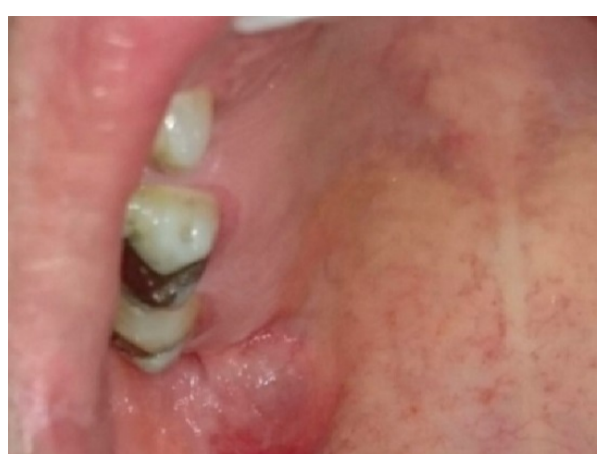

Fig. 1. Lesión en paladar, unilateral de 5 meses de evolución, asintomática con diagnósticos de trabajo: Mucocele vs CME

Colombia), refiriendo presencia de lesión asintomática en el paladar blando de 5 meses de evolución.

Al examen clínico presentó signos vitales dentro de los parámetros normales, sin adenomegalias cervicales ni cambios dérmicos.

Al examen clínico intraoral se observó lesión nodular de color violáceo a nivel de la unión de paladar duro con el paladar blando de lado derecho, de consistencia firme a la palpación, de $2 \mathrm{~cm}$ de diámetro mayor, estableciendo como impresión diagnostico Mucocele vs Carcinoma Mucoepidermoide.(Figura 1)

Se realizó biopsia escisional bajo anestesia local, obteniendo muestra de tejido cauchoso de color parduzco, la cual se envió en formol al $10 \%$, para ser procesada y realizar estudio histopatológico. (Figura 2)

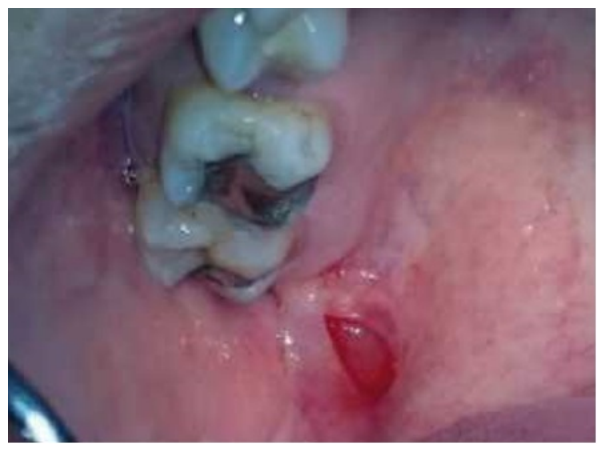

Fig. 2. Tejido cauchoso de color parduzco.

En el análisis de la biopsia enviada se observó a un campo de 40x la presencia de espacios quísticos con mucina en su interior, rodeados de células glandulares atípicas y con poca presencia de células epidermoides e intermedias. Por tanto el resultado del estudio histopatológico reportó Carcinoma Mucoepidermoide de bajo grado. (Figuras 3 y 4)

Se indicó exámenes complementarios, dentro de ellos Tomografía Axial Computarizada de cara simple y contrastada, exámenes de laboratorio (hemograma, BUN, creatinina, 


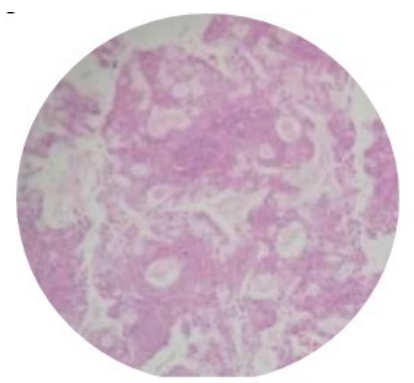

Fig. 3. (10X) Se observa pérdida de la arquitectura tisular y presencia de espacios quísticos con mucina en su interior

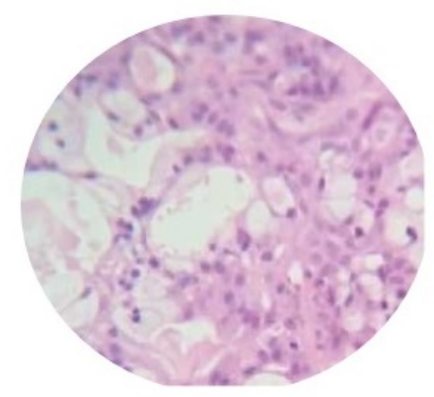

Fig. 4. (40x) Presencia de espacios quísticos con mucina en su interior, rodeados de células glandulares atípicas y con poca presencia de células epidermoides e intermedias

uroanálisis), radiografía de tórax.

En los cortes de la tomografía axial computarizada se observó una masa de densidad de tejido blando a nivel de la orofaringe comprometiendo el paladar blando derecho que cruza la línea media, de bordes irregulares, con diámetros mayores de 38x19mm, no se evidenció compromiso de tejido óseo; la orofaringe se preserva; las glándulas submandibulares y parótidas no ofrecieron alteración; no se observó adenomegalias de las cadenas cervicales, yugular y suprahioidea. (Figura 5)

La radiografía de tórax no mostró signos de metástasis tumoral y el hemograma mostró una ligera linfocitosis. Paciente es remitido a servicio de cabeza y cuello y al servicio de oncología del Hospital Central de la Policía Nacional para manejo.

\section{DISCUSIÓN}

Santos et al. ${ }^{10}$ manifiestaron que los CME de bajo grado son la variante más común y cursan como masas indoloras de morfología ovoide y tamaño inferior a $4 \mathrm{~cm}$. Igualmente Santos et al. ${ }^{10}$ refirió que es más frecuente entre la cuarta y la sexta década de vida, y afecta de forma similar a ambos sexos.

Por otro lado Liu et al. ${ }^{8}$ reportó que es más común en mujeres y en la quinta década de vida. Esto puede explicarse

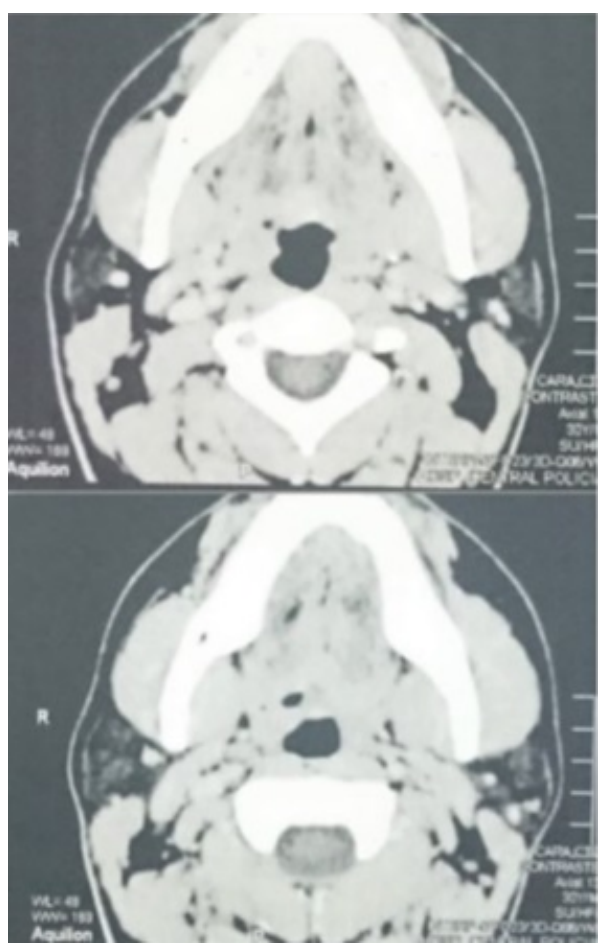

Fig. 5. Cortes de la tomografía axial computarizada.

por la muestra demográfica usada en cada estudio y en nuestro caso el paciente es de género masculino y en la cuarta década de vida lo que conincidió con lo manifestado por Santos et al. ${ }^{7}$ tal vez explicado por la cercanía geográfica entre Brasil y Colombia.

García et al. ${ }^{6}$ manifestaron que el Carcinoma Mucoepidermoide crece lentamente y tiene buen pronóstico, aunque puede producir metástasis. En nuestro caso la evolución de la neoplasia fue lenta (5 meses).

Chen et al. ${ }^{11}$ refirieron que la incidencia de afectación ganglionar cervical se presenta en un 3,3\% para el CME de bajo grado la supervivencia a los 5 años es del $90 \%$ y recurre el $6 \%$.

García et al y Ord et al ${ }^{6,12}$ tomaron como tratamiento de elección para Carcinomas Mucoepidermoide de bajo grado la resección quirúrgica con márgenes de seguridad, en nuestro caso la decisión por parte del servicio de Cirugía de Cabeza y Cuello fue la extirpación quirúrgica con márgenes de seguridad y valoración postquirúrgica del servicio de Oncología para Terapia coadyuvante.

En cuanto a lo que reporta la literatura, nuestro caso coincide en el cuadro clínico, morfología de la lesión, edad del paciente y manejo quirúrgico. Indicando la pertinencia de todas las acciones realizadas antes y durante el tratamiento 


\section{CONCLUSIÓN.}

Ante la presencia de lesiones en la cavidad bucal es importante contar con una amplia variedad de impresiones diagnósticas y realizar biopsia para establecer diagnóstico definitivo, con la finalidad de evitar consecuencias graves por un tratamiento tardío en caso de que se trate de lesiones malignas. La realización de una anamnesis y exploración detallada, acompañadas de exámenes complementarios es fundamental para establecer un diagnóstico y tratamiento adecuado.

Conflicto de intereses y financiamiento Los autores declaran no tener conflicto de intereses, haber cumplido con los requisitos de autoría y haber autofinanciado este artículo.

\section{Referencias}

1 Ávila, R. E., Samar, M. E., Fonseca, I., Olmedo, L., Asís, O. G., \&Ferraris, R. (2011). Carcinoma Mucoepidermoide de glándulas salivales: factores pronósticos histológicos e inmunohistoquímicos. International Journal of Morphology, 29(2), 455-462.

2 Passador, Santos, F., Brown, A., Kuasne, H., Soares, L. A., Lourenco, S., kowalskl, L.,\& Araujo, V. (2015). Gene Expression Profile of Mucoepidermoid Carcinoma of the salivary gland. Oral surgery, Oral Medicine, Oral Pathology and Oral Radiology, 120(3), e156.

3 Nakagaki, T., Ogi, K., Abe, M., Dehari, H., Miyazaki, A., Hasegawa, T., \& Hiratsuka, H. (2015). Low-grade mucoepidermoid carcinoma with regional lymph node metastasis: A case report and genetic review of criteria for grading. Journal of Oral and Maxillofacial Surgery, Medicine, and Pathology.

4 Rapidis, A. D., Givalos, N., Gakiopoulou, H., Stavrianos, S. D., Faratzis, G., Lagogiannis, G. A., \& Patsouris, E. (2007). Mucoepidermoid carcinoma of the salivary glands: Review of the literature and clinicopathological analysis of 18 patients. Oral oncology, 43(2), 130-136.

5 Troconis Ganimez, J. E. (2002). Carcinoma Mucoepidermoide: revisión de la literatura. Acta odontol. venez, 40(1), 43-46.

6 García-Molina, D., Castillo, J. L. D., Navarro, I., Sánchez, R., \& Burgueño, M. (2014). Carcinoma Mucoepidermoide de bajo grado: diagnóstico diferencial de masas palatinas. Revista Española de Cirugía Oral y Maxilofacial, 36(3), 136-137.

7 Santos, T. S., Melo, D. G., Andrade, E. S., Silva, E. D., \& Gomes, A. C. (2012). Carcinoma mucoepidermóide no palato: relato de caso. Revista Portuguesa de Estomatología, Medicina Dentária e Cirurgia Maxilofacial, 53(1), 29-33.

8 Liu, S., Ow, A., Ruan, M., Yang, W., Zhang, C., \& Wang, L. (2014). Prognostic factors in primary salivary gland mucoepidermoid Carcinoma: an analysis of 376 cases in an Eastern Chinese population. International journal of oral and maxillofacial surgery, 43(6), 667-673
9 Cruz Villca, M. B., Aillón López, H., \& Trujillo, C. (2007). Carcinoma Mucoepidermoide central: reporte de un caso. Cuaderno Hospital de Clínicas, 52, 87.

10 Santos P., Brown A., Kuasne H., Soares L., Lourenco S., KolwalskiL., Araujo V. (2015). Gene Expression Profile of Mucoepidermoid Carcinoma of the Salivary Gland. Oral Surgery, Oral Medicine, Oral Pathology and Oral Radiology, 120(3), e156.

11 Chen, M. M., Roman, S. A., Sosa, J. A., \& Judson, B. L. (2014). Histologic grade as prognostic indicator for mucoepidermoid carcinoma: A population-level analysis of 2400 patients. Head \& neck, 36(2), 158-163.

12 Ord, R. A., \& Salama, A. R. (2012). Is it necessary to resect bone for low-grade mucoepidermoid carcinoma of the palate?. British Journal of Oral and Maxillofacial Surgery, 50(8), 712-714.

Recibido: 16 de enero de 2017.

Aceptado: 20 de febrero de 2017. 